syndrome and were excluded. Of the 3 responders, 2 had the hyperactive-impulsive subtype of ADHD, and 1 had the ADD + HI combined type. All 3 had abnormal EEGs showing focal discharges in frontal areas. Two had received methylphenidate previously without benefit. ER-VPA was administered in one daily dose $(9.5$ to $18.8 \mathrm{mg} / \mathrm{kg}$ ) each morning. Serum VPA concentrations ranged from 26 to $58 \mathrm{mcg} / \mathrm{ml}$. Total ADHD-rating scale (RS-IV) scores were decreased following treatment with VPA, and the hyperactive-impulsive (H-I) symptoms were controlled more effectively than the inattentive type. Pretreatment and treatment scores for $\mathrm{H}-\mathrm{I}$ decreased from 26-11, 22-10, and 26-25, for patients 1,2, and 3, whereas those for inattention were 9-6, 9-9, and 24-21, respectively. SEP amplitudes following median nerve stimulation were decreased to normal voltage in two patients after VPA treatment. (Miyazaki M, Ito H, Saijo T, et al. Favorable response of ADHD with giant SEP to extended-release valproate. Brain Dev July 2006;28:470-472). (Respond: Dr Masahito Miyazaki, Department of Pediatrics, Miyoshi Medical Clinic, 813-1 Otani, Higashikagawa 769-2513, Japan).

COMMENT. The authors recommend a trial of ER-valproate in children with ADHD, especially those with the hyperactive-impulsive subtype, when associated with giant somatosensory evoked potentials. It should be noted that the responders also had epileptiform EEGs, with frontal localization, areas known to be involved in ADHD. Giant SEPs may reflect hyperactivity and decreased GABA in the sensorimotor cortex, and g-aminobutyric acid (GABA) enhancers such as clonazepam and valproate would be expected to reduce amplitude of giant SEPs and benefit hyperactive-impulsive behavior. In methylphenidate nonresponders, somatosensory evoked potentials should be tested, and treatment with VPA given consideration. GABAergic dysfunction as well as dopaminergic mechanisms are postulated in the etiology of ADHD. The risks of valproate therapy and the necessity for monitoring with blood level and other laboratory tests could deter its frequent use in ADHD practice.

Predictors for persistent hyperactive behavior in 2 to 7 year-olds included maternal prenatal smoking, child male gender, maternal depression, and hostile parenting. In a population-based sample, 7 children in 100 were classified as hyperactive at both the 2 -year and 7-year evaluations. Preventive intervention is recommended for high-risk families. (Romano E et al. Pediatrics June 2006;117:2101-2110).

\title{
TRIAL OF DEXMETHYLPHENIDATE (FOCALIN) ER IN ADHD
}

The efficacy and safety of dexmethylphenidate extended release (d-MPH-ER) was compared to placebo in 97 patients (ages 6-17 years) with attention-deficit/hyperactivity disorder (ADHD) in a multicenter, randomized, double-blind, two-phase study reported from New York State Psychiatric Institute, New York. The once daily dose of d-MPH-ER was flexible $(5-30 \mathrm{mg} / \mathrm{kg}$ ) for 5 weeks, and the patients' final optimal dose (mean, 24.0 +/- 7.1 $\mathrm{mg} /$ day) was maintained during the last 2 weeks of the 7-week trial. Conners ADHD/DSMIV Scale-Teacher version (CADS-T) total score improved significantly compared with placebo $(\mathrm{p}<.001)$, and $67.3 \%$ of patients were much improved on a Clinical Global Impressions-Improvement (CGI-I) Scale at final visit versus $13.3 \%$ of placebo patients $(\mathrm{p}<.001)$. Drug-related adverse events were spontaneously reported by $49.1 \%$ patients taking d-MPH-ER vs $25.5 \%$ of placebo-treated patients $(p=.01)$. Decreased appetite was drug- 
related in $28.3 \%$, headache in $9.4 \%$, and insomnia in $7.5 \%$. No patient discontinued the drug because of adverse events. (Greenhill LL, Muniz R, Ball RR, et al. Efficacy and safety of dexmethylphenidate extended-release capsules in children with attention-deficit/hyperactivity disorder. J Am Acad Child Adolesc Psychiatry July 2006;45:817-823). (Reprints: Dr Laurence L Greenhill, New York State Psychiatric Institute, 1051 Riverside Drive, Room 2307, New York, NY 10032).

COMMENT. At a mean daily dose of $24 \mathrm{mg} /$ day, d-MPH-ER was significantly superior to placebo in the treatment of ADHD. The mean dose of d-MPH-ER found effective is equivalent to a $50 \mathrm{mg} /$ day dose of racemic methylphenidate.

\section{BEHAVIORAL PREDICTORS OF SUBSTANCE-USE INITIATION IN ADOLESCENTS WITH AND WITHOUT ADHD}

Substance-use initiation was examined in 28 healthy adolescents and 50 with attention-deficit/hyperactivity disorder, followed longitudinally for 4 years at the National Institute of Mental Health, Bethesda, MD; National Institute on Drug Abuse, Baltimore, MD; and Neuropsychiatric Institute, Los Angeles, CA. Usage of tobacco, alcohol, and marijuana, substances most commonly reported, was not influenced by ADHD, ADHD and comorbid conduct disorder, or ADHD and depressive/anxiety. Among behavioral measures assessed at study entry, aggression was associated with tobacco smoking and marijuana use, and impulsivity with alcohol use. The severity of substance-use, indicated by the number of substances used, was correlated with and predicted by early aggressive behavior. (Ernst M, Luckenbaugh DA, Moolchan ET, et al. Behavioral predictors of substance-use initiation in adolescents with and without attention-deficit/hyperactivity disorder. Pediatrics June 2006;117:2030-2039). (Respond: Monique Ernst MD, PhD, Mood and Anxiety Disorders Program, National Institute of Mental Health, 15K North Dr, Room 118, MSC 2670, Bethesda, MD 20892).

COMMENT. A risk of using or abusing drugs in adolescence is a common concern of parents of children with ADHD, when discussing methylphenidate or other stimulant medication. The above report confirms previous studies showing that, except for impulsivity and alcohol, ADHD does not increase the risk of illicit substance-use or abuse in adolescence. A study at the Massachusetts General Hospital found drug abuse in $15 \%$ of 140 ADHD adolescents and the same frequency in 120 normal control subjects, followed for 4 years (Biederman J et al. 1997). The risk of alcohol and drug abuse was increased in patients with conduct or bipolar disorders, but not oppositional defiant disorder (ODD), major depression or anxiety. ODD, uncomplicated by conduct disorder did not predict drug abuse. In contrast to children and adolescents, adults with ADHD are more susceptible to drug abuse, especially if they have not previously received treatment for ADHD in childhood. Prescribed stimulant treatment for ADHD is found to decrease drug craving in adults with comorbid ADHD and drug abuse and to improve functioning (Horner BR, Scheibe KE, 1997). Psychosocial intervention and appropriate medications at an early age are important in prevention of substance abuse disorders in adults with pervasive ADHD. (Millichap JG. Attention Deficit Hyperactivity and Learning Disorders. Chicago, PNB Publishers, 1998) 